\title{
An Infinite Dimensional Approach to the Third Fundamental Theorem of Lie
}

\author{
Richard D. BOURGIN and Thierry P. ROBART \\ Department of Mathematics, Howard University, Washington DC 20059, USA \\ E-mail: rbourgin@howard.edu,trobart@howard.edu
}

Received November 02, 2007, in final form January 16, 2008; Published online February 16, 2008

Original article is available at http://www.emis.de/journals/SIGMA/2008/020/

\begin{abstract}
We revisit the third fundamental theorem of Lie (Lie III) for finite dimensional Lie algebras in the context of infinite dimensional matrices.

Key words: Lie algebra; Ado theorem; integration; Lie group; infinite dimensional matrix; representation

2000 Mathematics Subject Classification: 15A09; 15A29; 17A99; 17B66; 17D99; 22A22;

22A25; 22E05; 22E15; 22E45; 58H05
\end{abstract}

\section{Introduction}

At first blush the idea of revisiting a theorem from classical (i.e. finite dimensional) Lie group theory, no matter how pivotal, by pitching it in an infinite dimensional framework, may seem of questionable value. However, as we shall see, the cost of this more complicated context is mitigated by the restitution of absent, but long desired canonicity. This infinite dimensional setting, $\mathcal{M}(\infty)$, the space of infinite dimensional matrices, although the analog of the space $\mathcal{M}(n)$ of square matrices, provides a much richer and flexible environment in which to work, albeit with additional subtleties and complexities. The embedding construction we will present for formal power series leads us to the appropriate concept of invertibility in $\mathcal{M}(\infty)$.

Recall that the third fundamental problem of Lie is today understood as being the integrability question of a given Lie algebra into a differential group or local group. Ado's celebrated theorem [1,2] asserts that any finite dimensional Lie algebra admits a faithful finite dimensional matrix representation. This result provides an easy algebraic procedure for integrating any Lie algebra into a (matrix) Lie group without needing the Frobenius theorem. However, classical matrix representations turn out to be quite limited. Indeed, there are finite dimensional Lie groups with no faithful finite dimensional matrix representations (see [3] for a modern characterization of matrix Lie groups).

This finite dimensional limitation may be circumvented by introducing infinite dimensional matrices. To reconstruct a given Lie group we can start from its set of right invariant vector fields. The infinite setting allows to encapsulate not only its Lie algebra structure but also its underlying finer analytic structure; it offers a way to restore in the large the above mentioned easy integration procedure.

After recalling Cartan's and Olver's versions of Lie's third fundamental theorem (Lie III) (Section 2) we describe how to embed the basic tools of classical differential geometry into matrix form (Section 3.1) and offer a brief survey of the main definitions and properties of $\mathcal{M}(\infty)$ (Section 3.2). Finally, we illustrate (Lie III) in this new setting (Section 4.1). We get, as

\footnotetext{
*This paper is a contribution to the Proceedings of the Seventh International Conference "Symmetry in Nonlinear Mathematical Physics" (June 24-30, 2007, Kyiv, Ukraine). The full collection is available at http://www.emis.de/journals/SIGMA/symmetry2007.html
} 
consequence, that all finite dimensional Lie groups, as well as local Lie groups, admit an infinite dimensional matrix representation. Technical details will be published in a forthcoming paper [6].

\section{The third fundamental theorem}

Let us first recall the well-known global version of Lie's third fundamental theorem [7].

Theorem 1 (Lie III - Élie Cartan). Each Lie algebra $\mathcal{L}$ of finite dimension is the Lie algebra of a unique (up to isomorphism) connected and simply connected Lie group $\tilde{G}$. Moreover any connected Lie group $G$ having $\mathcal{L}$ as Lie algebra is a quotient of that simply connected Lie group $\tilde{G}$ by a discrete normal subgroup of its center.

We now recall a less known but still fundamental version of (Lie III). This quite recent version is due to Peter Olver [12]. It concerns the integrability question of local Lie groups. Not all local Lie groups can be embedded into a (global) Lie group! Before stating its main theorem we follow [12] in illustrating his construction through a basic example.

Let $G=\mathbb{R}^{2}$ denote the additive 2-dimensional real Lie group and let $S=\{(-1,0)\} \subset G$. Denote by $M=G \backslash S$ the corresponding punctured plane. Observe that the original global action of $G$ on itself (by translation) naturally gives rise to a local action of $G$ on $M$. Finally, construct a simply connected smooth covering space $L$ of $M$ with projection map $\pi: L \longrightarrow M$. Since $\pi$ is a local isomorphism, we may lift the local action of $G$ on $M$ to $L$. This allows us to regard the space $L$ as a topological space of mostly invertible elements containing an identity element. In fact all but the pre-image by $\pi$ of $S^{-1}=-S=\{(1,0)\}$ are invertible elements with inverses in $L$.

More precisely, let us start with the following definition [12, Definition 2] of a local Lie group.

Definition 1 (Local Lie group). A smooth manifold $L$ is called a local Lie group if there exists

a) a distinguished element $e \in L$, the identity element,

b) a smooth product map $\mu: \mathcal{U} \rightarrow L$ defined on an open subset $\mathcal{U}$ satisfying

$$
(\{e\} \times L) \cup(L \times\{e\}) \subset \mathcal{U} \subset L \times L,
$$

c) a smooth inversion map $\iota: \mathcal{V} \rightarrow L$ defined on an open subset $\mathcal{V}, e \in \mathcal{V} \subset L$ such that $\mathcal{V} \times \iota(\mathcal{V}) \subset \mathcal{U}$, and $\iota(\mathcal{V}) \times \mathcal{V} \subset \mathcal{U}$, all satisfying the following properties:

(i) identity: $\mu(e, x)=x=\mu(x, e)$ for all $x \in L$,

(ii) inverse: $\mu(\iota(x), x)=e=\mu(x, \iota(x))$ for all $x \in \mathcal{V}$,

(iii) associativity: if $(x, y),(y, z),(\mu(x, y), z)$ and $(x, \mu(y, z))$ all belong to $\mathcal{U}$, then $\mu(x, \mu(y, z))$ equals $\mu(\mu(x, y), z)$.

With this definition in mind it is possible - see [12] - to select two open sets $\mathcal{U}$ and $\mathcal{V}$ of the covering space $L$ of $M$ to endow $L$ with the structure of a local Lie group. Interestingly, $L$, like most local Lie groups, cannot be enlarged into a global Lie group. The crucial observation to be made is that local associativity (assumption (iii)) may not be extended to global associativity. To see this, consider the points $\mathbf{a}=(-2,1), \mathbf{b}=(0,-2)$ and $\mathbf{c}=(2,1)$ in $M$. Start at the origin $\mathbf{0}$ of $M$ acting first through translation by $t \mathbf{a}$ for $t \in[0,1]$ then, starting at $\mathbf{a}$, acting through translation by $t \mathbf{b}$ for $t \in[0,1]$, and finally, starting at $\mathbf{a}+\mathbf{b}$, acting through translation by $t \mathbf{c}$ for $t \in[0,1]$. Of course we end up back at the origin since $\mathbf{a}+\mathbf{b}+\mathbf{c}=\mathbf{0}$. The triangle $\Delta$ we have just described contains the singular point $(-1,0)$. Let us mimic this procedure on $L$ with the corresponding lifted actions, starting at the identity element of $L, \overline{\mathbf{0}}$, in the $0^{\text {th }}$ sheet of $L$. Denote 
by $\overline{\mathbf{a}}, \overline{\mathbf{b}}, \overline{\mathbf{c}}$ the lifted elements on the $0^{\text {th }}$ sheet of $L$ of $\mathbf{a}, \mathbf{b}$ and $\mathbf{c}$. Then $\overline{\mathbf{a}}+(\overline{\mathbf{b}}+\overline{\mathbf{c}})=\overline{\mathbf{0}}$ as may be checked directly. In particular, this sum lies in the $0^{\text {th }}$ sheet of $L$. But $(\overline{\mathbf{a}}+\overline{\mathbf{b}})+\overline{\mathbf{c}} \neq \overline{\mathbf{a}}+(\overline{\mathbf{b}}+\overline{\mathbf{c}})$ since the left side lies in the first sheet of $L$, not the $0^{\text {th }}$. In other words, global associativity has been broken!

It was P.A. Smith and A.I. Mal'cev who first pointed out in the 1930's $[14,15,10]$ the crucial connection between globalizability and associativity. It took sixty more years to obtain a general method for constructing local Lie groups which are not contained in any global Lie group [12]. The local Lie group $L$ constructed above offers a nice illustration of Olver's general construction:

Theorem 2 (Lie III - Peter Olver). Let $G$ be a connected Lie group and $S \subset G$ a closed subset of $G$ not containing the identity. Any covering manifold $\pi: L \longrightarrow G \backslash S$ can be regarded as a local Lie group. Moreover each local Lie group is contained in one of these examples.

\section{Infinite dimensional matrices}

There is a plethora of equivalent formats for the set $\mathcal{M}(\infty)$ of infinite (countable) dimensional matrices. Among them the format $\mathbb{N} \times \mathbb{N}$ (with $\mathbb{N}=\{1,2, \ldots\}$ the set of positive integers and starting from the upper-left corner) distinguishes itself for its simplicity. This is the format we shall focus on throughout this paper.

\subsection{Embedding tools}

\subsubsection{The fundamental tools of differential geometry}

Differential geometry makes a crucial use of the Lie pseudo-groups of smooth diffeomorphisms in $n$ variables. Their infinite jets give rise to the groupoids $\Gamma_{n}$ of formal transformations (power series) in $n$-variables. For simplicity let us focus here on the one dimensional case. The general case will be presented in [6].

Let $\Gamma_{\mathbb{R}}$ be the Lie groupoid of formal transformations of the real line [16]. Thus

$$
\Gamma_{\mathbb{R}}=\left\{g=\sum_{k=0}^{+\infty} a_{k}(x-p)^{k} \mid a_{k}, p \in \mathbb{R}, a_{1} \neq 0\right\}
$$

endowed with the double bundle

$$
\mathbb{R} \stackrel{s}{\longleftarrow} \Gamma_{\mathbb{R}} \stackrel{t}{\longrightarrow} \mathbb{R}
$$

for which the source map $s$ is defined by $s(g)=p$, and the target map $t$ by $t(g)=a_{0}$. Observe that $x$ in $g=\sum_{k=0}^{+\infty} a_{k}(x-p)^{k}$ is a dummy variable which does not really need to be mentioned. It will be useful at times to make such a choice explicit. We will then write $g(x)$ instead of just $g$ to emphasize that choice.

Suppose $g_{i}, i=1,2$ belong to $\Gamma_{\mathbb{R}}$. Their product $g_{1} \cdot g_{2}$ is defined only when $t\left(g_{2}\right)=s\left(g_{1}\right)$, and in this case $g_{1} \cdot g_{2}=g_{1} \circ g_{2}$, the formal composition of series. Note that the restriction $a_{1} \neq 0$ in the definition of $g \in \Gamma_{\mathbb{R}}$ guarantees that the formal Bürmann-Lagrange series (see [9] for example) for $g^{-1}$ exists and is in $\Gamma_{\mathbb{R}}$. Of course, for $g=\sum_{k>0} a_{k}(x-p)^{k} \in \Gamma_{\mathbb{R}}$, we have $s\left(g^{-1}\right)=t(g)=a_{0}$ and $t\left(g^{-1}\right)=s(g)=p$. Evidently, it is not possible in general to express $g$ ( or $g^{-1}$ ) as a power series about any point other than its source. Hence, in particular, we usually have to move the center of coordinates to go from the power series expansion of $g$ to that of $g^{-1}$ when $p \neq a_{0}$. This elementary observation makes it clear why, in the representation of $\Gamma_{\mathbb{R}}$ in the space of infinite matrices (see below), the inverse of a given matrix may not be expressible in the same system of coordinates as is the matrix itself. Indeed, a change of basis is required most of the time! 


\subsubsection{Matrix representation of $s^{-1}(0)$}

There are infinitely many ways to represent in matrix form $s^{-1}(0)$, i.e. the formal (power series) transformations based at the origin of the system of coordinates. The procedure we follow is very natural.

For $g \in s^{-1}(0) \subset \Gamma_{\mathbb{R}}$ and for each positive integer $m$, let $g(x)^{m}$ denote the $m$-fold product (as opposed to composition) of $g=g(x)$ with itself and let $u_{g(x)}$ be the column vector

$$
u_{g(x)}=\left(\begin{array}{lllll}
1 & g(x) & g(x)^{2} & g(x)^{3} & \ldots
\end{array}\right)^{T},
$$

where ${ }^{T}$ denotes transpose. Note that, with $g$ the identity transformation, we get the column vector

$$
u_{x}=\left(\begin{array}{lllll}
1 & x & x^{2} & x^{3} & \ldots
\end{array}\right)^{T} .
$$

Finally, denote by $M_{g}$ the coefficient matrix of the power series representations of the entries in $u_{g(x)}$ centered at 0 . Thus

$$
M_{g} u_{x}=u_{g(x)} .
$$

Then $g \stackrel{\rho}{\mapsto} M_{g}$ is a faithful algebraic representation of $s^{-1}(0)$ in $\mathcal{M}(\infty)$. For $g_{1}, g_{2} \in s^{-1}(0)$, their composition $g_{1} \circ g_{2}$ (defined when $t\left(g_{2}\right)=0$ ) corresponds to matrix multiplication. Moreover, if $t\left(g_{1}\right)=0$ and $t\left(g_{2}\right)=0$ then both $M_{g_{1}}$ and $M_{g_{2}}$ are upper triangular. Otherwise said, through the infinite dimensional linear representation $\rho$, the isotropy group of the transitive Lie groupoid $\Gamma_{\mathbb{R}}$ is a subgroup of the nice (formal) group of upper triangular matrices [4].

Consider now the element $g=1-x+x^{2}+\cdots+(-1)^{n} x^{n}+\cdots$ of $\Gamma_{\mathbb{R}}$. Note that $g \in s^{-1}(0)$, and that its associated matrix is

$$
M_{g}=\left(\begin{array}{cccccccc}
1 & 0 & 0 & 0 & 0 & 0 & 0 & \ldots \\
1 & -1 & 1 & -1 & 1 & -1 & 1 & \cdots \\
1 & -2 & 3 & -4 & 5 & -6 & 7 & \\
1 & -3 & 6 & -10 & 15 & -21 & 28 & \\
1 & -4 & 10 & -20 & 35 & -56 & 84 & \\
1 & -5 & 15 & -35 & 70 & -126 & 210 & \\
\vdots & \vdots & & & & & & \ddots
\end{array}\right)
$$

Since $g=\tau \circ h$ where $\tau(x)=1+x$ and $h(x)=-x+x^{2}-x^{3}+\cdots, M_{g}$ decomposes as $M_{g}=M_{\tau} M_{h}$ with

$$
M_{\tau}=\left(\begin{array}{cccccccc}
1 & 0 & 0 & 0 & 0 & 0 & 0 & \ldots \\
1 & 1 & 0 & 0 & 0 & 0 & 0 & \ldots \\
1 & 2 & 1 & 0 & 0 & 0 & 0 & \\
1 & 3 & 3 & 1 & 0 & 0 & 0 & \\
1 & 4 & 6 & 4 & 1 & 0 & 0 & \\
1 & 5 & 10 & 10 & 5 & 1 & 0 & \\
\vdots & \vdots & & & & & & \ddots
\end{array}\right) \quad \text { and } \quad M_{h}=\left(\begin{array}{cccccccc}
1 & 0 & 0 & 0 & 0 & 0 & 0 & \ldots \\
0 & -1 & 1 & -1 & 1 & -1 & 1 & \ldots \\
0 & 0 & 1 & -2 & 3 & -4 & 5 & \\
0 & 0 & 0 & -1 & 3 & -6 & 10 & \\
0 & 0 & 0 & 0 & 1 & -4 & 10 & \\
0 & 0 & 0 & 0 & 0 & -1 & 5 & \\
\vdots & \vdots & & & & & & \ddots
\end{array}\right)
$$

Each element of $\Gamma_{\mathbb{R}}$ is invertible as a groupoid element. In particular, $g$ has an inverse in $\Gamma_{\mathbb{R}}$ given explicitly by $h^{-1} \circ \tau^{-1}$. While both $\left(M_{\tau}\right)^{-1}=M_{\tau^{-1}}$ and $\left(M_{h}\right)^{-1}=M_{h^{-1}}=M_{h}$ exist individually as matrices, we observe that their product $\left(M_{h}\right)^{-1}\left(M_{\tau}\right)^{-1}$ does not make sense (i.e. some infinite series in the matrix product do not converge). In other words, the geometric candidate for $\left(M_{g}\right)^{-1}$ fails to be "visible" (i.e. convergent) in the present chart. We are led to enlarge the classical notion of invertibility. 


\subsubsection{The associated groupoids - their matrix form}

In the previous paragraphs we have discussed a matrix representation for the elements of the source fiber $s^{-1}(0)$ of $\Gamma_{\mathbb{R}}$. Let us extend this representation to the entire groupoid $\Gamma_{\mathbb{R}}$. Observe that if $g \in \Gamma_{\mathbb{R}}$ then $g=\tau_{1} \circ \gamma \circ \tau_{2}$ where $\gamma \in s^{-1}(0) \cap t^{-1}(0)$ (i.e. $\gamma$ is in the isotropy group of $\left.\Gamma_{\mathbb{R}}\right)$ and $\tau_{1}, \tau_{2}$ are translations given explicitly by $\tau_{1}(x)=t(g)+x$ and $\tau_{2}(x)=-s(g)+x$. As pointed out above (in the special case of the translation $x \longrightarrow 1+x$ ), both $\tau_{1}$ and $\tau_{2}$ have matrix representations as unipotent lower triangular matrices, and $\gamma$ as an upper triangular matrix. Hence each element of $\Gamma_{\mathbb{R}}$ has a matrix representation as a formal product $L_{1} U \star L_{2}$ in which $L_{i}, i=1,2$ are lower triangular and $U$ is upper triangular. The star here represents a latent (undone) matrix product.

Remark 1. The above mentioned latent product is not always convergent if carried out. When it is we lose crucial information by performing it: the knowledge of the source of the corresponding groupoid element. Doing so would be equivalent to prolonging an analytic germ in a neighborhood of its point of definition and then extracting the germ of the constructed section at another point. On the contrary this remark does not hold for the other product $L_{1} U$. In other terms, $L_{1} U$ is equivalent to $L_{1} \star U$. This is in itself quite surprising!

\subsection{Invertible matrices}

We just observed that the classical concept of invertibility (existence of an inverse) needs to be enlarged in order to accept in the infinite dimensional context most geometrical constructions. In this section we describe the appropriate concept together with some important properties. See [5] for more details.

\subsubsection{Which matrices are really invertible?}

In most of what follows the choice $\mathbb{R}$ or $\mathbb{C}$ for scalar field is immaterial, and when it is, it is denoted $\mathbb{K}$. As already described, by an infinite matrix we shall mean an array $A=\left(a^{i}{ }_{j}\right)$ in which the indices $i$ and $j$ run over the positive integers, $\mathbb{N}$, the upper index denoting the row and the lower, the column. Let $E^{0}$ be the vector space of column vectors with finitely many nonzero entries indexed by $\mathbb{N}$ whose entries are in $\mathbb{K}$. Thus $E^{0}$ is the inductive limit of the $\mathbb{K}^{n}$ with the natural injections.

Definition 2 ( $\gamma$-invertibility). A matrix $A=\left(a^{i}{ }_{j}\right)$ is $\gamma$-invertible if both $A$ and $A^{T}$ have trivial kernel as linear maps defined on $E^{0}$.

Thus $A$ is $\gamma$-invertible if and only if both its set of columns and its set of rows are linearly independent (for finite linear combinations). Matrices invertible in the classical sense - i.e. that admit two-sided inverses - are $\gamma$-invertible. Moreover, for upper - or lower - triangular matrices, it is straightforward to check that the $\gamma$ - and classical notions of invertibility coincide, and each is equivalent to having all nonzero diagonal entries. These are straightforward consequences of the definition. In the next section we describe some useful, not so obvious consequences.

\subsubsection{Main properties of $\gamma$-invertible matrices}

Our goal here is to provide a glimpse into the rich structure of the space of $\gamma$-invertible matrices. The $P L U$-decomposition theorem provides justification for the definition, and is a very useful characterization of such matrices. The concept of $\beta$-block mentioned below is a useful notion in embedding groupoids of transformations in more than one variable, like $\Gamma_{\mathbb{R}^{n}}$ or groupoids of formal generalized symmetries of systems of partial differential equations [13]. 
Recall that a permutation matrix can be characterized as follows. It is filled with 0 s and $1 \mathrm{~s}$ and each of its columns and rows contains a unique 1 .

Theorem 3 (PLU-decomposition). Each $\gamma$-invertible matrix A admits a PLU decomposition. Here $P$ denotes a permutation matrix, $L$ a lower triangular unipotent matrix and $U$ an invertible upper-triangular matrix. Conversely any such product PLU is a $\gamma$-invertible matrix.

The connectivity of the set of $\gamma$-invertible matrices is in stark contrast to its finite dimensional counterpart.

Theorem 4 (Connectedness). The set $\mathcal{M}^{\star}$ of $\gamma$-invertible matrices with real entries and indexed by $\mathbb{N}$ is arcwise connected in the Tychonov topology. The same holds if the index set $\mathbb{N}$ for matrix indices is replaced by $\mathbb{Z}$, or $\mathbb{R}$ by $\mathbb{C}$.

As in the finite dimensional case we can characterize $\gamma$-invertible matrices using determinants. Indeed, let $\pi=\left(\pi_{1}, \pi_{2}\right)$ denote an ordered pair of permutations of $\mathbb{N}$. For any $M \in \mathcal{M}(\infty)$ denote by $\Delta_{n}^{\pi}(M)$ the determinant of the $n \times n$ submatrix of $M$ with rows $\pi_{1}(1), \ldots, \pi_{1}(n)$ and columns $\pi_{2}(1), \ldots, \pi_{2}(n)$. Let $\beta: \mathbb{N} \hookrightarrow \mathbb{N}$ be an increasing injection. Define the $\beta$-block $\sigma$-determinant of $M$ associated with $\pi$ as the sequence $\Delta_{\beta}^{\pi}(M)=\left\{\Delta_{\beta(n)}^{\pi}(M)\right\}_{n \in \mathbb{N}}$. Say that this $\beta$-block $\sigma$-determinant is nonzero on $M$, when $\Delta_{\beta(n)}^{\pi}(M) \neq 0$ for all $n$. When $\beta=\operatorname{id}_{\mathbb{N}}\left(\mathrm{id}_{\mathbb{N}}\right.$ is the identity map on $\mathbb{N}$ ) suppress the subscript and write more simply $\Delta^{\pi}(M)=\left\{\Delta_{n}^{\pi}(M)\right\}_{n \in \mathbb{N}}$, the $\sigma$-determinant of $M$ associated with $\pi$.

Theorem 5 (Characterization of $\gamma$-invertibility by $\boldsymbol{\sigma}$-determinants). Let $M \in \mathcal{M}$. The following are equivalent

(i) $M$ is $\gamma$-invertible;

(ii) there exists a pair $\pi, \beta$ such that $\Delta_{\beta}^{\pi}(M) \neq 0$;

(iii) there exists $\pi_{1}$ such that, with $\pi_{2}=i d_{\mathbb{N}}, \Delta^{\pi}(M) \neq 0$;

(iv) there exists $\pi_{2}$ such that, with $\pi_{1}=i d_{\mathbb{N}}, \Delta^{\pi}(M) \neq 0$.

\section{Revisiting Lie's third theorem}

\subsection{An algebraic approach}

In order to reformulate (Lie III, Élie Cartan), recall that the quotient of a connected Lie group $G$ by a discrete normal subgroup of its center $N$ gives rise to a covering $\pi: G \longrightarrow G / N$ where $\pi$ is a (locally one-to-one) group homomorphism. This puts, in a neighborhood of the identity, both versions of Lie's third fundamental theorem (see Section 2) on the same footing. The restriction of the covering map to an appropriate (small enough) neighborhood $\bar{U}$ of the identity of the covering (local) group always leads to an isomorphism of local Lie groups

$$
\pi: \bar{U} \subset L \longrightarrow U \subset G \text {. }
$$

Recall moreover that all (local) Lie groups possess a natural compatible real analytic manifold structure. Following the line of argument in Section 3, we may represent any local analytic transformation in $\gamma$-invertible matrix form. This leads to

Theorem 6 (Matrix representation). Each finite dimensional Lie group or local Lie group can be faithfully represented in $\mathcal{M}^{\star}$, the set of $\gamma$-invertible matrices. In this representation, group composition is represented by matrix multiplication which always turns out to be locally absolutely convergent. 
The detailed proof will be published in [6]. It involves in an essential way the real analytic manifold structure of (local) Lie groups, the existence of a canonical chart of the first kind for local Lie groups and the Morrey-Grauert embedding theorem of analytic manifolds into a Euclidean space $[11,8]$. The above mentioned covering structure admits, in our context, a counterpart involving the adjoint action [6].

\subsection{Illustration}

Let us represent $(\mathbb{R},+)$ among the $\gamma$-invertible matrices and, by using an appropriate adjoint action, map it locally isomorphically onto the unit circle $S^{1}$. For $a, z \in \mathbb{C}$ denote by $\tau_{a}$ translation by $a$ - that is, $\tau_{a}(z)=a+z$. Denote its matrix form by $T_{a}$. Thus if

$$
u_{z}=\left(\begin{array}{c}
1 \\
z \\
z^{2} \\
z^{3} \\
\vdots
\end{array}\right) \quad \text { then } \quad \tau_{a}(z) \leftrightarrow T_{a} u_{z}=u_{z+a}
$$

where

$$
T_{a}=\left(\begin{array}{ccccc}
1 & & & & \\
a & 1 & & & \\
a^{2} & 2 a & 1 & & \\
a^{3} & 3 a^{2} & 3 a & 1 & \\
\vdots & & & & \ddots
\end{array}\right)
$$

(Note that we are now working in $\Gamma_{\mathbb{C}}=\Gamma_{\mathbb{R}} \otimes \mathbb{C}$.) The embedding schema is as follows:

$$
z \stackrel{\ln }{\longrightarrow} \tilde{z} \stackrel{\tau_{i y}}{\longrightarrow} \tilde{z}+i y \stackrel{\exp }{\longrightarrow} z e^{i y}
$$

which transforms, by adjoint action, $\mathbb{R}$ (identified here with $\{i y \mid y \in \mathbb{R}\}$ ) onto $S^{1}$. In fact, an interval of $\mathbb{R}$ centered at 0 is transformed in this way onto a neighborhood of $1 \in S^{1}$. Finally, the extension of this local group isomorphism to the local isomophism it induces from $(\mathbb{R},+)$ onto $S^{1}$ proceeds by extension, step by step, increasing the domains on which the local group operations are defined, until objects are produced which are closed under both the group operation and inversion. The details follow.

To start, using the embedding of $\Gamma_{\mathbb{R}}$ in the space of infinite dimensional matrices already discussed, let the matrix forms of the functions $z \rightarrow \ln (1+z)$ and $z \rightarrow-1+\exp (z)$, the isotropy part of exp, be denoted with capital letters. Thus, within the domains of absolute convergence of the power series representations centered at 0 of these analytic maps,

$$
\mathrm{LN}=\left(\begin{array}{cccccc}
1 & 0 & 0 & 0 & 0 & \cdots \\
& 1 & -1 / 2 & 1 / 3 & -1 / 4 & \\
& & 1 & -1 & 11 / 12 & \\
& & & 1 & -3 / 2 & \\
& & & & 1 & \\
& & & & & \ddots
\end{array}\right) \quad \text { and } \quad \mathrm{EXP}_{0}=\left(\begin{array}{cccccc}
1 & 0 & 0 & 0 & 0 & \ldots \\
0 & 1 & 1 / 2 ! & 1 / 3 ! & 1 / 4 ! & \\
0 & 0 & 1 & 1 & 4 / 3 & \\
0 & 0 & 0 & 1 & 3 / 2 & \\
0 & 0 & 0 & 0 & 1 & \\
\vdots & & & & & \ddots
\end{array}\right) .
$$

These matrices satisfy the defining equations $\mathrm{LN} u_{z}=u_{\ln (1+z)}$ and $\operatorname{EXP}_{0} u_{z}=u_{-1+\exp (z)}$. Thus in matrix notation, the above scheme may be written

$$
u_{z} \longrightarrow T_{-1} u_{z} \longrightarrow\left(T_{i y} \mathrm{LN}\right)\left(T_{-1} u_{z}\right) \longrightarrow T_{1}\left[\mathrm{EXP}_{0}\left(T_{i y} \mathrm{LN}\right)\right]\left(T_{-1} u_{z}\right)
$$


Since these matrix multiplications correspond, faithfully, to the composition of the corresponding functions, in order to understand in which regions we will have absolute convergence, we may (and do) concentrate on the functions themselves. Thus, for example, within the radius of absolute convergence,

$$
\begin{aligned}
& \left(\operatorname{EXP}_{0}\right)\left(T_{i y}(\mathrm{LN})\right) \leftrightarrow\{z \longrightarrow \underbrace{i y+\left(z-\frac{z^{2}}{2 !}+\frac{z^{3}}{3 !}-\frac{z^{4}}{4 !}+\cdots\right)}_{\omega}+\frac{\omega^{2}}{2 !}+\frac{\omega^{3}}{3 !}+\cdots\} \\
& =\left(e^{i y}-1\right)+z\left(e^{i y}\right) \\
& +z^{2}\left[-\frac{1}{2}+\frac{1}{2 !}(1-i y)+\frac{1}{3 !}\left(3 i y-\frac{3}{2}(i y)^{2}\right)+\frac{1}{4 !}\left(6(i y)^{2}-\frac{(i y)^{3}}{2} \cdot 4\right)+\cdots\right] \\
& +z^{3}[\cdots]+\cdots=\left(e^{i y}-1\right)+z\left(e^{i y}\right) .
\end{aligned}
$$

It follows that

$$
T_{1}\left[\operatorname{EXP}_{0}\left(T_{i y} \mathrm{LN}\right)\right] T_{-1} \leftrightarrow e^{i y}+(z-1) e^{i y}=z e^{i y} .
$$

That is, the adjoint action $\pi(i \mathbb{R}) \pi^{-1}\left(\right.$ where $\pi(i y)=e^{i y}$ ) sends y to the scaling function: $z \rightarrow$ $z e^{i y}$. Of course, in matrix form this function has the representation

$$
\left(\begin{array}{cccccc}
1 & 0 & 0 & 0 & 0 & \cdots \\
0 & e^{i y} & 0 & 0 & 0 & \\
0 & 0 & e^{2 i y} & 0 & 0 & \\
0 & 0 & 0 & e^{3 i y} & 0 & \\
0 & 0 & 0 & 0 & e^{4 i y} & \\
\vdots & & & & & \ddots
\end{array}\right)
$$

which may be identified with $e^{i y} \in S^{1}$. These calculations presuppose that all the relevant infinite series converge absolutely. Note that LN is applied to $T_{-1} u_{z}$ so the function version is $\ln (1+(z-1))$. Hence the condition for absolute convergence is $|z-1|<1$. For each fixed $y \in \mathbb{R}$, the portion of $S^{1}$ for which $|z-1|<1$ is the arc of $S^{1}$ between polar angles $-\pi / 3$ and $\pi / 3$. Using this representation of $i y$ as the above diagonal matrix, it is easy to expand the domain of $y$ 's for which iy may be represented, by multiplication of matrices. After one set of multiplications we will have an association between $\{i y \mid y \in(-2 \pi / 3,2 \pi / 3)\}$, and within two more expansions the circle will be completely covered by an interval of $\mathbb{R}$.

We could similarly embed Olver's basic example (see Section 2) in matrix form. Indeed, it suffices to observe that $L$ identifies with the Riemann surface associated to $z \rightarrow \ln (1+z)$.

\subsection{Perspectives}

Let $G$ denote the one parameter complex group generated by the infinite matrix $X$ displayed below. Thus $G=\{\exp (t X) \mid t \in \mathbb{C}\}$. By way of an elementary example we will show how an adjoint action can alter $G$ in ways not possible in the finite dimensional setting. Take $X$ to be the matrix with nonzero entries only in the first column given explicitly by

$$
X=\left(\begin{array}{rrrrr}
0 & & & & \cdots \\
-1 & 0 & & & \\
1 & & 0 & & \\
-1 & & & 0 & \\
\vdots & & & \ddots
\end{array}\right) .
$$


Let

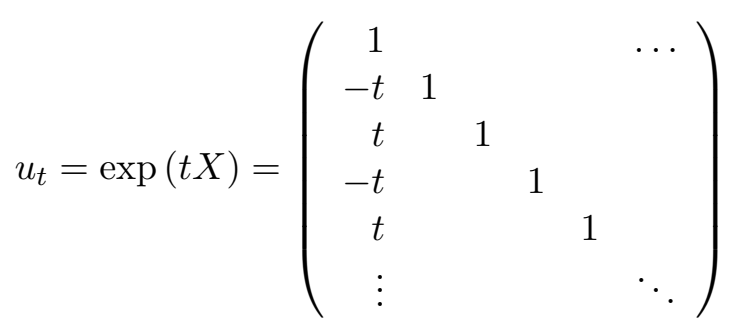

and take

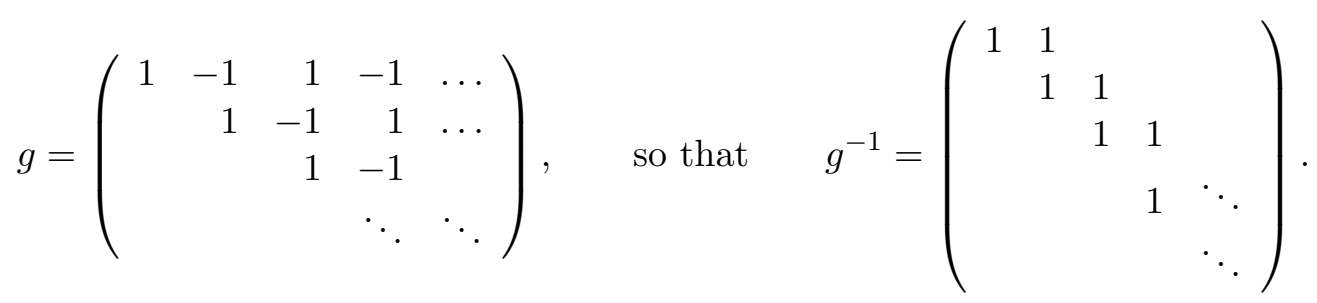

Then $\operatorname{Ad}(g) G=\left\{g^{-1} u_{t} g \mid t \in \mathbb{C}\right\}$. If $M_{t}$ denotes $g^{-1} u_{t} g$ then by direct calculation,

$$
M_{t}=\left(\begin{array}{cccccc}
1-t & t & -t & t & -t & \ldots \\
0 & & & & & \\
0 & & & & & \\
0 & & & I & & \\
0 & & & & &
\end{array}\right)
$$

in which the identity matrix $I$ appears starting in the second row and second column.

Observe that

$$
\begin{aligned}
& M_{t} M_{t^{\prime}}=\left(\begin{array}{cccccc}
1-t & t & -t & t & -t & \ldots \\
0 & 1 & 0 & 0 & 0 & \\
0 & 0 & 1 & 0 & 0 & \\
0 & 0 & 0 & 1 & 0 & \\
0 & 0 & 0 & 0 & 1 & \\
\vdots & & & & & \ddots
\end{array}\right)\left(\begin{array}{cccccc}
1-t^{\prime} & t & -t^{\prime} & t^{\prime} & -t^{\prime} & \ldots \\
0 & 1 & 0 & 0 & 0 & \\
0 & 0 & 1 & 0 & 0 & \\
0 & 0 & 0 & 1 & 0 & \\
0 & 0 & 0 & 0 & 1 & \\
\vdots & & & & & \ddots
\end{array}\right) \\
& =\left(\begin{array}{cccccc}
(1-t)\left(1-t^{\prime}\right) & (1-t) t^{\prime}+t & -(1-t) t^{\prime}-t & (1-t) t^{\prime}+t & -(1-t) t^{\prime}-t & \ldots \\
0 & 1 & 0 & 0 & 0 & \\
0 & 0 & 1 & 0 & 0 & \\
0 & 0 & 0 & 1 & 0 & \\
0 & 0 & 0 & 0 & 1 & \\
\vdots & & & & & \ddots
\end{array}\right) \\
& =M_{t+t^{\prime}-t t^{\prime}} .
\end{aligned}
$$

That is, addition of complex numbers: $\left(t, t^{\prime}\right) \rightarrow t+t^{\prime}$ has been transformed into

$$
\left(t, t^{\prime}\right) \rightarrow \mu\left(t, t^{\prime}\right)=t+t^{\prime}-t t^{\prime} .
$$

Observe that $M_{t}$ is $\gamma$-invertible unless $t=1$. Hence the domain of $\mu$ is no longer $\mathbb{C} \times \mathbb{C}$, but instead $(\mathbb{C} \backslash\{1\}) \times(\mathbb{C} \backslash\{1\})$. It is easy to check that $\left\{M_{t} \mid t \in \mathbb{C} \backslash\{1\}\right\}$ is a group under matrix multiplication isomorphic to $(\mathbb{C} \backslash\{1\}, \mu)$. In particular, $\mu$ is associative.

It is remarkable to observe that the adjoint action $\operatorname{Ad}(g)$ of this example has immediately altered - without offering any convergence problem - the topology of the group. In this case $\mathbb{C}$ has been transformed into $\mathbb{C} \backslash\{1\}$. 


\section{References}

[1] Ado I.D., Note on the representation of finite continuous groups by means of linear substitutions, Izv. Fiz.-Mat. Obsch. (Kazan) 7 (1935), 1-43 (in Russian).

[2] Ado I.D., The representation of Lie algebras by matrices, Transl. Amer. Math. Soc. (1) 9 (1962), 308-327.

[3] Beltitua D., Neeb K.-H., Finite-dimensional Lie subalgebras of algebras with continuous inversion, math.FA/0603420.

[4] Bochner S., Formal Lie groups, Ann. of Math. 47 (1946), 192-201.

[5] Bourgin R., Robart T., Generalization of the concept of invertibility for infinite dimensional matrices, submitted.

[6] Bourgin R., Robart T., On Ado's theorem, in preparation.

[7] Cartan É., La Topologie des Groupes de Lie, Exposés de Géométrie No. 8, Hermann, Paris, 1936.

[8] Grauert H., On Levi's problem and the imbedding of real-analytic manifolds, Ann. of Math. 68 (1958), 460-472.

[9] Henrici P., Applied and computational complex analysis, Vol. 1, Wiley, 1974.

[10] Malcev A.I., Sur les groupes topologiques locaux et complets, C. R. Acad. Sci. URSS 32 (1941), 606-608.

[11] Morrey C.B., The analytic embedding of abstract real-analytic manifolds, Ann. of Math. 68 (1958), $159-201$.

[12] Olver P.J., Non-associative local Lie groups, J. Lie Theory 6 (1996), 23-51.

[13] Robart T., About the local and formal geometry of PDE, Contemp. Math. 285, (2001), $183-194$.

[14] Smith P.A., Topological foundations in the theory of continuous transformation groups, Duke Math. J. 2 (1936), 246-279.

[15] Smith P.A., Topological groups, in Proceedings of the International Congress of Mathematicians (1950, Cambridge, Mass.), Vol. 2, Amer. Math. Soc., Providence, R.I., 1952, 436-441.

[16] Weinstein A., Groupoids: unifying internal and external symmetry, Notices Amer. Math. Soc. 43 (1996), 744-752, math.RT/9602220. 